\title{
Loss of $\alpha$-actinin-3 during human evolution provides superior cold resilience and muscle heat generation
}

Wyckelsma VL ${ }^{1 *}$, Venckunas $T^{2 *}$, Houweling $\mathbf{P J}^{3,4}$, Schlittler $\mathbf{M}^{1}$, Lauschke $\mathbf{V M}^{1}$, Tiong $\mathbf{C F}^{3,4}$, Wood $\mathbf{H}^{3,4}$, Ivarsson $\mathbf{N}^{1}$, Paulauskas $\mathbf{H}^{2}$, Eimantas $\mathbf{N}^{2}$, Andersson DC ${ }^{1,5}$, North $\mathbf{K N}^{3,4}$, Brazaitis $\mathbf{M}^{2 \#}$, Westerblad $\mathbf{H}^{1,2 \#}$

* equal contribution; \# shared senior authorship

1. Department of Physiology and Pharmacology, Karolinska Institutet, Stockholm, Sweden

2. Institute of Sport Science and Innovations, Lithuanian Sports University, Kaunas,

2 Lithuania

3. Murdoch Children's Research Institute, The Royal Children's Hospital, Melbourne, Australia

4. Department of Paediatrics, University of Melbourne, Melbourne, Australia

5. Heart and Vascular Theme, section for Heart Failure, Arrhythmia and GUCH, Karolinska University Hospital, Stockholm, Sweden

Corresponding Author:

Prof. Håkan Westerblad Department of Physiology and Pharmacology Biomedicum C5 Karolinska Institutet 17177 Stockholm, Sweden hakan.westerblad@ki.se 


\section{ABSTRACT}

The fast skeletal muscle protein $\alpha$-actinin-3 is absent in 1.5 billion people worldwide due to homozygosity for a nonsense polymorphism in the ACTN3 gene (R577X) ${ }^{1}$. The prevalence of the $577 \mathrm{X}$ allele increased as modern humans moved to colder climates, suggesting a link between $\alpha$-actinin-3 deficiency and improved cold tolerance ${ }^{1,2}$. Here, we show that humans lacking $\alpha$-actinin-3 (XX) are superior in maintaining core body temperature during coldwater immersion due to changes in skeletal muscle thermogenesis. Muscles of $\mathrm{XX}$ individuals displayed a shift towards more slow-twitch isoforms of myosin heavy chain (MyHC) and sarcoplasmic reticulum (SR) proteins, accompanied by altered neuronal muscle activation resulting in increased tone rather than overt shivering ${ }^{3,4}$. Experiments on Actn3 knockout mice showed no alterations in brown adipose tissue (BAT) properties that could explain the improved cold tolerance in XX individuals. Thus, this study provides a clear mechanism for the positive selection of the ACTN3 X-allele in cold climates and supports a key thermogenic role of skeletal muscle during cold exposure in humans.

\section{Main}

The sarcomeric protein $\alpha$-actinin-3 resides in the Z-discs of fast skeletal muscle fibers, where it cross-links the actin filaments of adjacent sarcomeres ${ }^{5-7}$. The lack of functioning ACTN3 does not cause muscle disease, but it has been shown to affect muscle function both in the general population and in athletes ${ }^{8,9}$; in general, $\alpha$-actinin-3 deficiency is detrimental for power and sprint activities ${ }^{8,10,11}$.

A study exploring evolutionary implications of $\alpha$-actinin-3 deficiency demonstrated that the X-allele became more abundant as humans migrated out of Africa into the colder climates of central and northern Europe ${ }^{1,2,12}$. This led to the hypothesis that $\alpha$-actinin-3 deficient humans are superior in adapting to lower temperature ${ }^{2}$. On this basis, we sought to determine whether $\alpha$-actinin-3 deficient (XX) humans were better at defending their body temperature during an acute cold challenge than humans with functioning ACTN3 (RR). Young male XX and RR individuals (Supplementary Table 1) were immersed in $14{ }^{\circ} \mathrm{C}$ water for 20 min periods interposed by $10 \mathrm{~min}$ pauses in room-tempartured air; cold-water exposure was continued until the rectal temperature reached $35.5^{\circ} \mathrm{C}$ or for a total of $120 \mathrm{~min}(170 \mathrm{~min}$ including the pauses). The percentage of individuals able to maintain their body temperature above $35.5^{\circ} \mathrm{C}$ degrees for the complete cold-water exposure was markedly higher in the XX group (69\%) than in the RR group (30\%) (Fig. 1A). The average rate of decline of rectal $\left(\mathrm{T}_{\mathrm{re}}\right)$ and gastrocnemius muscle $\left(\mathrm{T}_{\mathrm{mu}}\right)$ temperatures in $\mathrm{XX}$ subjects was about half of that in $\mathrm{RR}$ 
60 subjects (Fig. 1B and 1C). The rate of skin temperature $\left(\mathrm{T}_{\mathrm{sk}}\right)$ decline, on the other hand, was 61 not significantly different between the two groups (Fig. 1D). The overall increase in energy 62 consumption induced by the cold challenge was assessed by measurements of heart rate and 63 the rate of respiratory $\mathrm{O}_{2}$ uptake $\left(\mathrm{VO}_{2}\right)$ and $\mathrm{CO}_{2}$ exhalation $\left(\mathrm{VCO}_{2}\right)$; all three rates were 64 significantly increased at the end of cold exposure irrespective of ACTN3 genotype (Fig. 1E65 G). Thus, $\alpha$-actinin-3 deficient individuals showed superior protection of core body 66 temperature during an acute cold stress and this was achieved without increased energy 67 consumption as judged from similar cold-induced increases in heart rate, $\mathrm{VO}_{2}$ and $\mathrm{VCO}_{2}$ in $68 \quad \mathrm{XX}$ and RR individuals. 


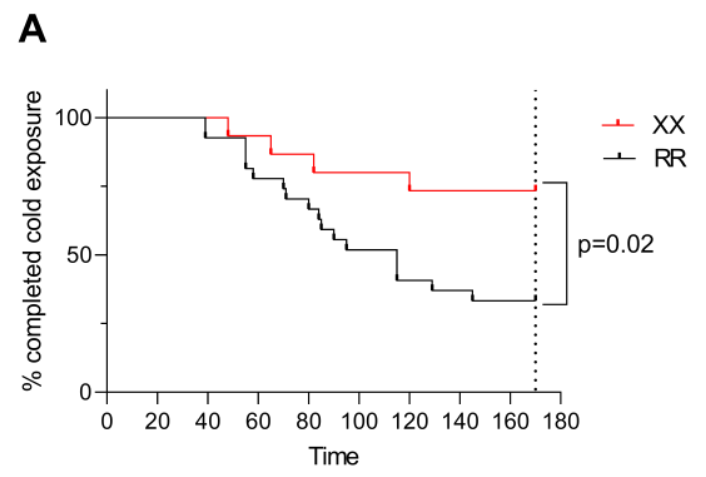

B

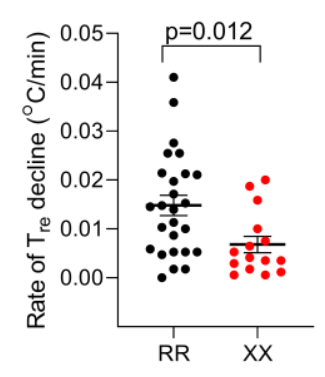

E

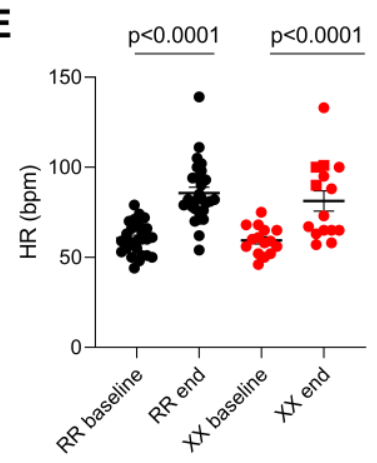

C
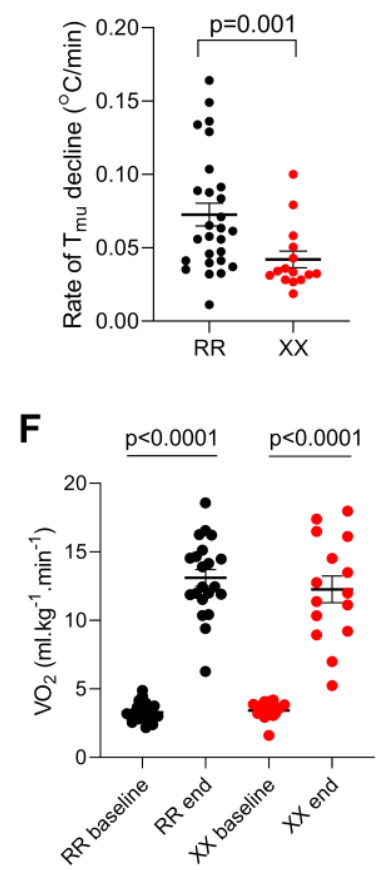

D

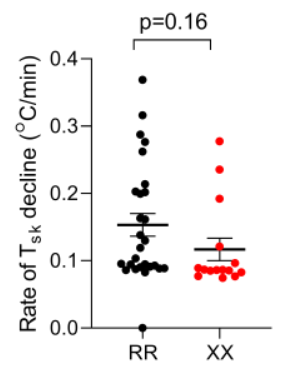

G

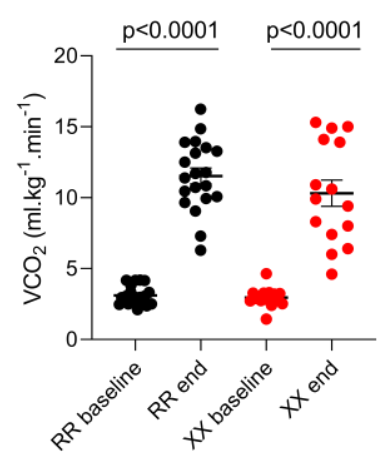

Fig. 1. Temperature measurements and physiological responses during cold-water

immersion. A) Survival plot of the time taken to reach a rectal temperature $\left(T_{r e}\right)$ of $35.5{ }^{\circ} \mathrm{C}$ or sustaining the complete 170 min period of cold-water immersion in $R R(n=27)$ and $X X(n$ = 15) individuals. Log-rank (Mantel-COX) test was used to assess statistical difference between $R R$ and $X X$ individuals. The decline rate in rectal $\left(T_{r e} ; \boldsymbol{B}\right)$, intramuscular $\left(T_{m u} ; \boldsymbol{C}\right)$ and skin $\left(T_{s k} ; \boldsymbol{D}\right)$ temperatures in RR and XX subjects. Statistical difference between the two groups was assessed with unpaired t-test. Heart rate $(\boldsymbol{E})$ and rate of pulmonary $\mathrm{O}_{2}$ uptake $\left(\mathrm{VO}_{2}, \boldsymbol{F}\right)$ and $\mathrm{CO}_{2}$ exhalation $\left(\mathrm{VCO}_{2}, \boldsymbol{G}\right)$ before (baseline) and at the end of cold-water immersion in $R R$ and $X X$ subjects. Statistical assessment with 2-way RM ANOVA revealed no differences between the two groups either before or at the end of cold-water exposure. Plots show values for each RR (black circles) and XX (red circles) individual and mean \pm SEM. 
In mice, Actn3 knockout (KO) results in changes in intracellular $\mathrm{Ca}^{2+}$ handling with marked increases in $\mathrm{SR} \mathrm{Ca}^{2+}$ leak and the subsequent heat-generating active $\mathrm{SR} \mathrm{Ca}^{2+}$ re-uptake via the SR $\mathrm{Ca}^{2+}$-ATPase (SERCA) ${ }^{13}$. Uncoupling of SERCA activity from the actual $\mathrm{Ca}^{2+}$ transport into the SR is considered a key component in muscular non-shivering thermogenesis ${ }^{14-17}$. SERCA is expressed in several different isoforms in mammalian tissues with SERCA1 and SERCA2a being the main isoforms in adult fast-twitch and slow-twitch muscle fibers, respectively ${ }^{18-20}$. Notably, we detected a shift in dominance from SERCA1 in RR muscles to SERCA2a in XX muscles (Fig. 2A-B). The SR $\mathrm{Ca}^{2+}$ storage protein, calsequestrin (CSQ), also shows a fiber type-dependent isoform distribution with CSQ1 dominating in fast-twitch fibers and CSQ2 in slow-twitch fibers ${ }^{19,21}$. We observed similar total CSQ expression in XX and RR muscles, whereas the expression of CSQ2 was about twice as high in XX compared to RR muscles (Fig. 2C-D). The difference in SERCA and CSQ isoform expression between XX and RR muscles may reflect a larger volume of the muscle to be composed of slow-twitch fibers in XX than in RR subjects. Therefore, we used high-sensitivity silver staining to analyze the MyHC composition and found that XX muscles had significantly more slow MyHC I ( $\beta$-MyHC) and less fast MyHC IIx than RR muscles (Fig. 2E).

The sarcomeric $\alpha$-actinins are known to interact with a multitude of functionally

101 diverse proteins involved in structural, metabolic, signaling, and $\mathrm{Ca}^{2+}$-handling pathways ${ }^{10}$.

102 We used proteomics as an exploratory measure to look for further differences in muscle between XX and RR individuals. This proteomic analysis of skeletal muscle biopsies shows distinct differences in protein abundance between XX and RR individuals. Overall, we found 42 proteins to be differentially expressed between genotypes (Fig. 2F), including higher protein levels of slow-twitch muscle fiber markers (MyHC I (MYH7), slow-type myosin-

107 binding protein C (MYBPC1), SERCA2) and lower levels of fast-twitch markers (myosin 108 light chain 1/3 (MYL1), myosin regulatory light chain 2 (MYLPF), fast-type muscle troponin $109 \mathrm{~T}$ (TNNT3)) in XX than in RR individuals (Fig. 2G). Furthermore, pathway analysis revealed 110 that differentially expressed proteins were enriched in pyruvate metabolism $(P=0.0003$; 111 FDR = 0.02), including pyruvate dehydrogenase (PDHA1) with an $\sim 60 \%$ higher protein 112 expression in $\mathrm{XX}$ than in RR individuals. 

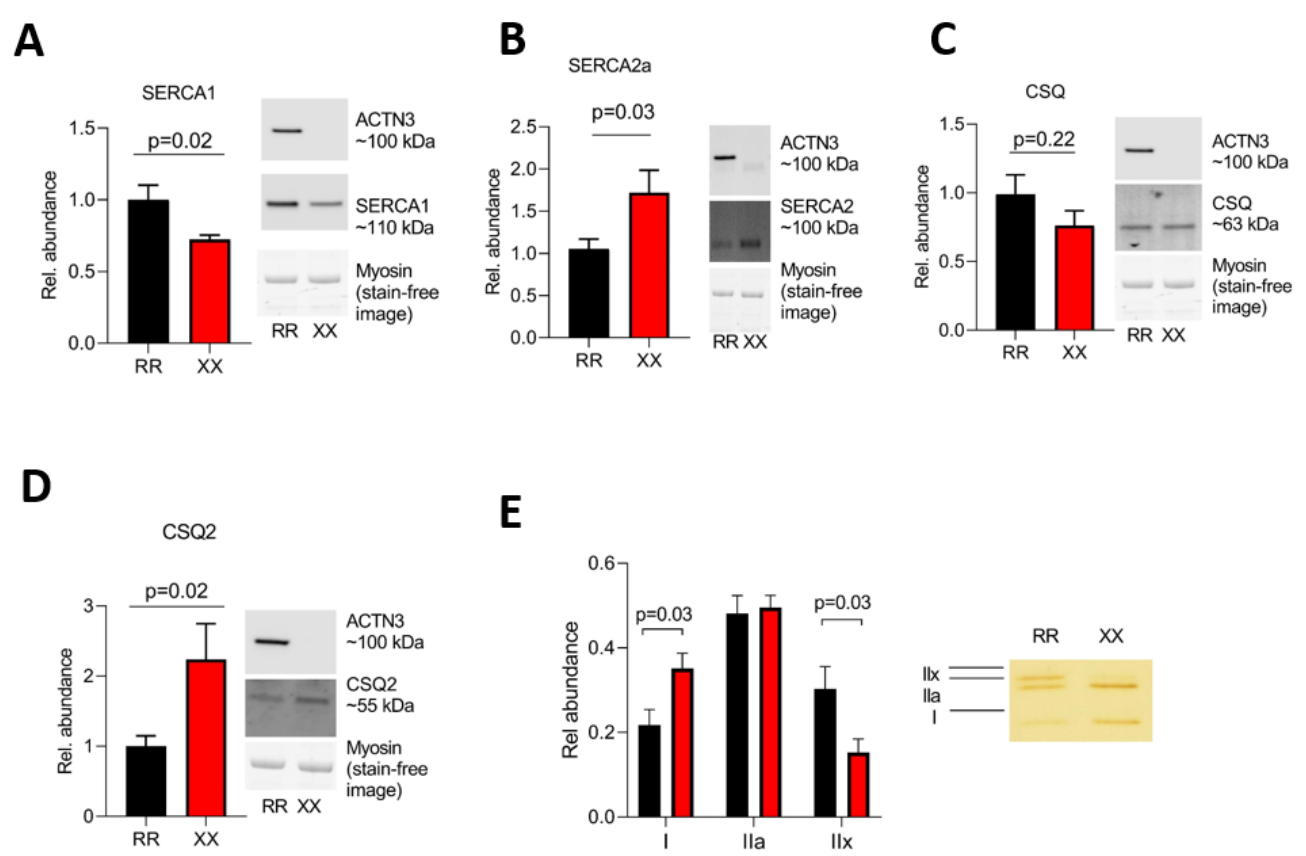

$\mathbf{E}$
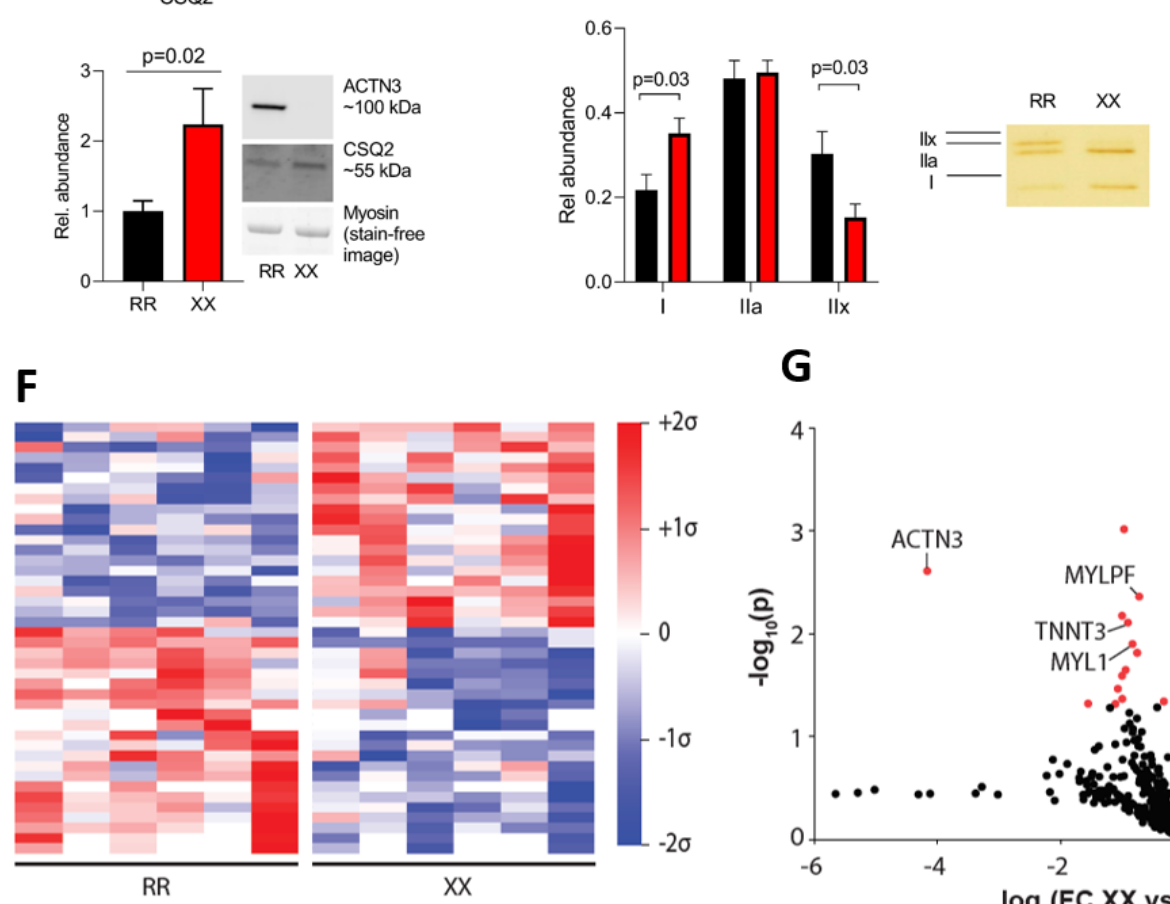

G

115 Fig. 2. ACTN3 deficiency is accompanied by a shift towards a slower skeletal muscle

116 phenotype. Summary data (mean \pm SEM) and representative western blots of the SR $\mathrm{Ca}^{2+}$ -

117 handling proteins SERCA1 (A), SERCA2a $(\boldsymbol{B}), \operatorname{CSQ}(\boldsymbol{C})$, and CSQ2 $(\boldsymbol{D})$ in muscle of $R R(n=$

118 8) and $X X(n=7)$ individuals. Band intensities were normalized to their respective myosin

119 loading controls. Data expressed relative to the mean value of the RR group, which was set to

120 1.0. Statistical difference between the two groups was assessed with unpaired t-test. E) Silver

121 stained gels were used to assess the distribution of MyHC isoforms in $R R(n=7)$ and XX $(n$

122 = 7) individuals. Right part shows a representative example of the distribution of MyHC in

$123 R R$ and XX individuals. The total staining of the three MyHC bands was set to 1 in each

124 subject. Statistical difference between the two groups was tested with unpaired t-test. $\boldsymbol{F}$ )

125 Mean-centered sigma-normalized heatmap of differentially expressed proteins $(P<0.05)$. $G)$

126 Volcano plot of all identified proteins $(n=601)$ expressed as fold-change $(F C)$ in $X X$

127 compared to $R R$ individuals. Differentially abundant proteins are indicated in red. 
129 Although the difference in SERCA and CSQ isoform expression between XX and RR muscle

130 homogenates corresponds with the MyHC distributions, $\alpha$-actinin-3 deficiency might still 131 affect the abundance of these $\mathrm{Ca}^{2+}$-handling proteins within individual fiber types. However, 132 fiber type-specific western blots performed on pooled single muscle fibers did not reveal any 133 fiber type-dependent differences in the expression of SERCA1, total CSQ or CSQ2 between $134 \mathrm{XX}$ and RR muscles (Supplementary Fig. 1). Intriguingly, SERCA2a was higher in the RR than in XX Type I fibers, which might indicate that the abundance of SERCAs is not the limiting factor in temperature regulation. SERCA might be involved in muscular nonshivering thermogenesis via its associated protein sarcolipin (SLN), which interferes with SERCA function by mediating uncoupling of the $\mathrm{SR} \mathrm{Ca}^{2+}$ uptake from the heat-generating ATP hydrolysis ${ }^{15,22}$. Hence, we measured SLN expression in muscle homogenates and in pooled single fibers, and the results showed no difference in SLN expression between XX and RR muscles (Supplementary Fig. 2 and Supplementary Fig. 3). Another potential $\mathrm{Ca}^{2+}$ SR-related mechanism for heat generation is $\mathrm{Ca}^{2+}$ leak through the $\mathrm{SR}^{2} \mathrm{Ca}^{2+}$ release channel, the ryanodine receptor 1 (RyR1) channel complex, due to dissociation of the channelstabilizing subunit FK506 binding protein (FKBP12) ${ }^{23,24}$. However, immunoprecipitation experiments did not reveal any difference in the amount of FKBP12 bound to RyR1 between XX and RR muscles (Supplementary Fig. 2B). To summarize, we could not detect any SR $\mathrm{Ca}^{2+}$-handling protein-dependent explanation for the superior cold tolerance of $\mathrm{XX}$ subjects. Brown adipose tissue is an important heat-producing thermo-effector in mammals ${ }^{25}$. However, adult humans have relatively little brown adipose tissue (BAT) and it is therefore difficult to assess its role as a heat generator ${ }^{26}$. We utilized the well-defined Actn3 KO mouse model to examine the impact of BAT activation as a mechanism for improved heat generation in $\alpha$-actinin-3 deficient individuals ${ }^{1}$. Wildtype (WT) and Actn3 KO mice were kept in a cold $\left(4{ }^{\circ} \mathrm{C}\right)$ room for 5 hours and core body temperature was measured at regular intervals using a rectal probe. In accordance with the human results, the number of mice able to maintain their body temperature above $35.5^{\circ} \mathrm{C}$ for the cold exposure period was markedly higher in the Actn3 KO (41\%) than in the WT (16\%) group (Fig. 3A). Although the overall rate of temperature decline during cold exposure was not significantly different between the two groups (Fig. 3B), Actn3 KO mice were significantly lighter than their WT counterparts (Fig. 3C) and therefore showed an improved cold tolerance after normalizing for body weight

160 (Fig. 3D). Following cold exposure, Actn3 mRNA was present in BAT of WT mice, but 161 absent in the KO mice (Fig. 3E). RNA-sequencing was performed on BAT collected from 
162 mice subjected to either thermal neutrality $\left(\mathrm{TN}, 30^{\circ} \mathrm{C}\right)$, room temperature $\left(\mathrm{RT}, 22{ }^{\circ} \mathrm{C}\right)$ or low 163 temperature (cold, $4{ }^{\circ} \mathrm{C}$ ). An unbiased principal component analysis (PCA) and heat map 164 clustering separated samples based on temperature (TN, RT and cold), whereas it showed no 165 effect of Actn3 genotype (Fig. 3F and G). Further analysis showed over 2000 differentially 166 expressed genes in BAT following cold exposure (Fig. 3H); however, only one transcript was 167 significantly altered based on genotype and temperature (cold; glycine/arginine rich protein 168 1; Grrp1), excluding physiologically relevant genotype-specific differences in BAT 169 properties following acute cold exposure (Fig. 3I). To sum up, these data imply that the 170 improved cold tolerance observed in $\alpha$-actinin- 3 deficient humans and mice is due to 171 improved skeletal muscle heat generation without any detectable influence of BAT. 
A

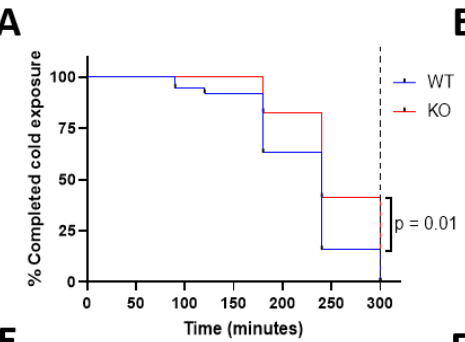

E

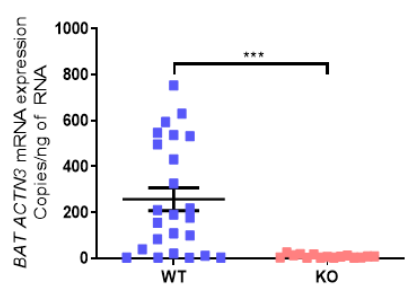

H

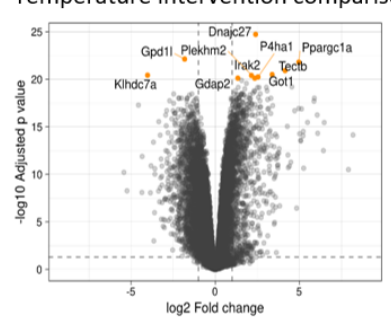

B

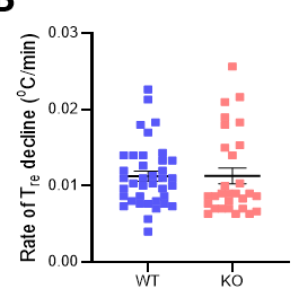

$\mathbf{F}$
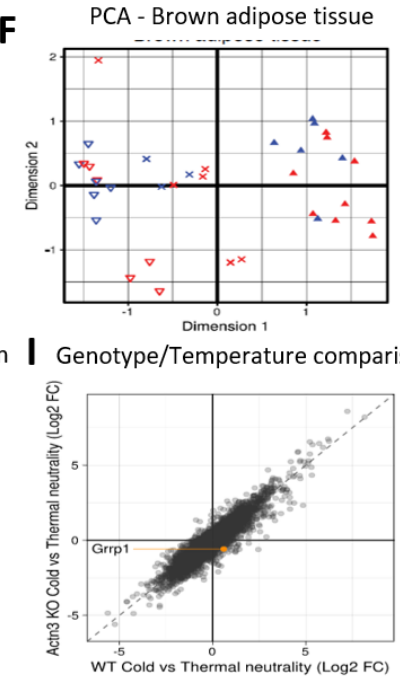

C
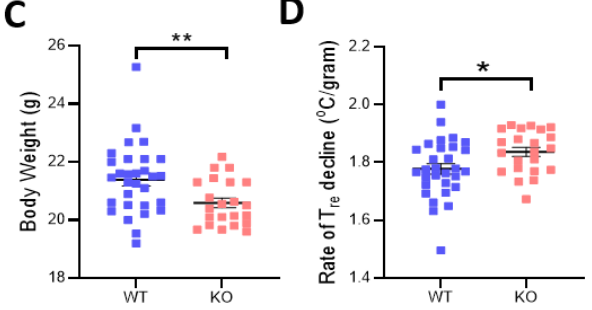

G
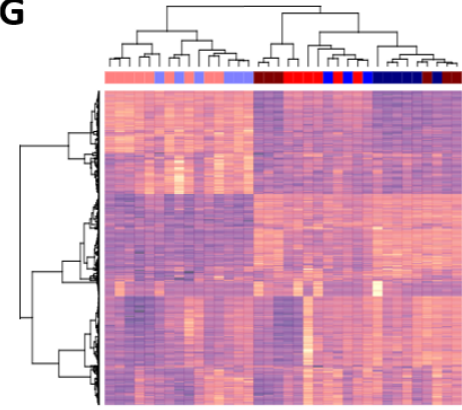
\begin{tabular}{r|r|r} 
Thermal neutrality & \\
\hline Room temperature &
\end{tabular}

cold exposure $\square$

Figure 3: Improved cold tolerance in Actn3 KO mice not related to altered BAT properties. A) Survival plot of the time taken to reach $35.5^{\circ} \mathrm{C}$ core body temperature over a 5 hours period exposed to $4^{\circ} \mathrm{C}$ air temperature in WT $(n=38)$ and Actn3 KO $(n=29)$ mice. Log-rank (Mantel-COX) test was used to assess statistical difference between WT and KO mice. The overall rate of decline in rectal body temperature $(\boldsymbol{B})$, body weight $(\boldsymbol{C})$ and rate of rectal body temperature decline per g body weight (D). E) Following cold exposure, Actn3 mRNA is present in WT but not in Actn3 KO mice. RNA-sequencing analyses show an effect of temperature but no effect of Actn3 genotype on BAT activation with both the principal component analysis $(P C A, \boldsymbol{F})$ and heat map $(\boldsymbol{G})$. $\boldsymbol{H})$ Volcano plot of altered genes confirms that marked changes in BAT gene expression occurs following acute cold exposure, with >2000 differentially expressed genes identified. I) Interaction plot shows no differences

187 based on Actn3 genotype in BAT following acute cold exposure. $* P<0.05$, **P<0.01, ***P $<0.001$ with unpaired t-test. 
190 The major heat-generating mechanism in mammalian skeletal muscle during an acute cold

191 challenge is involuntary activation of motor units resulting in skeletal muscle contraction.

192 This mechanism is generally referred to as shivering thermogenesis although it involves both

193 increased basal muscle tone due to continuous low-intensity activation and overt shivering

194 due to high-intensity bursting activity ${ }^{3}$. In our human cohort, we used surface

195 electromyography (EMG) to follow the activation of pectoralis major muscles during cold-

196 water immersion and observed more frequent bursting activity in RR individuals with mean

197 data showing an approximately two times higher rate of bursts in RR than in XX muscles

198 (Fig. 4A and B); the markedly higher activity during bursts also resulted in a slightly higher

199 mean EMG signal frequency in RR muscles (Fig. 4C). On the other hand, there was no

200 significant difference in the amplitude of the EMG signal, which reflects the overall number

201 of muscle fibers being activated (Fig. 4D). Interestingly, cold-induced low-intensity

202 continuous muscle activity is associated with activation of type I muscle fibers, whereas high-

203 intensity burst activity is linked to recruitment of type II muscle fibers ${ }^{4,27,28}$. Thus, the

204 difference in burst rate between XX and RR muscles is consistent with the difference in fiber

205 type distribution with more type I and less type II MyHC in XX than in RR muscles (see Fig.

206 2E). 
A

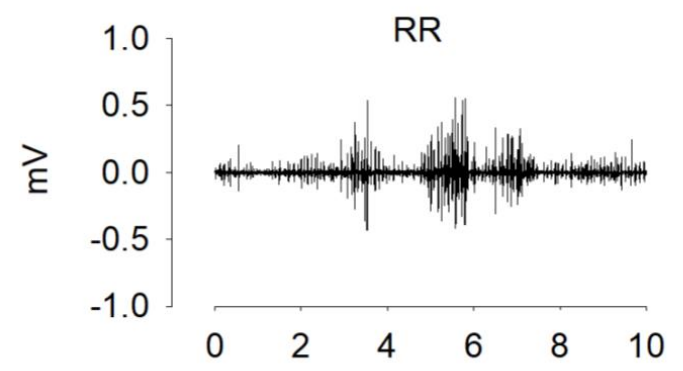

$X X$

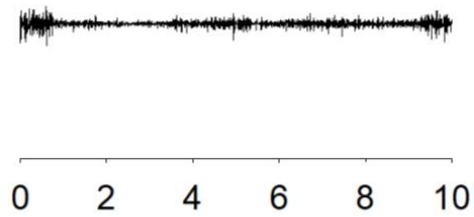

Time (s)
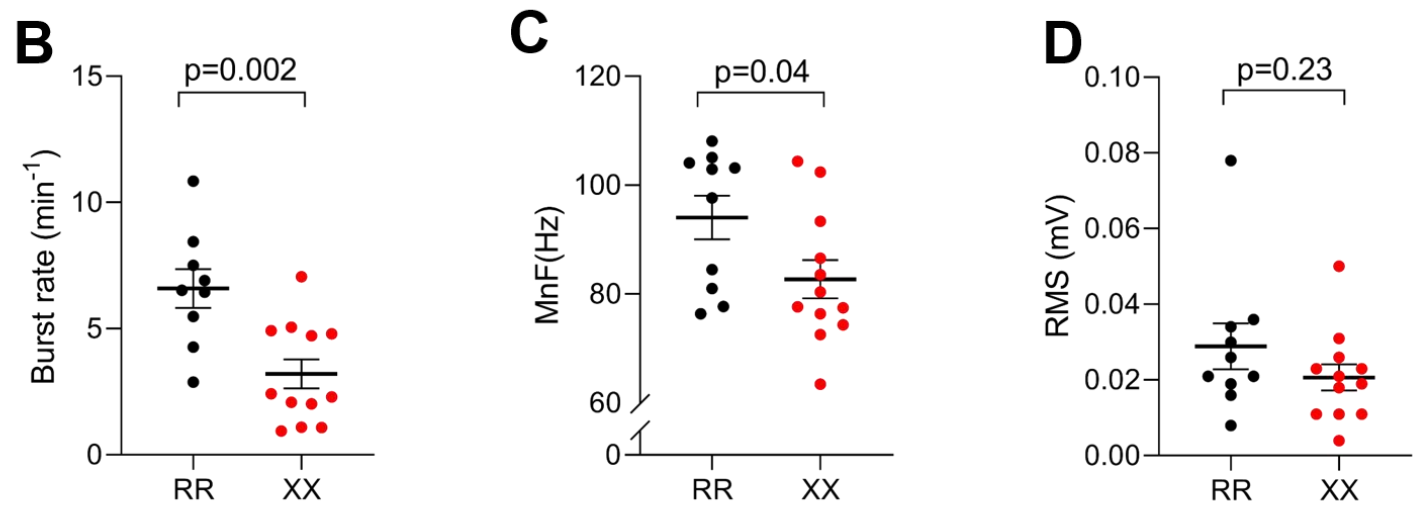

210 Figure 4. Bursting muscle activity rather than increased muscle tone is more prominent in

$211 \boldsymbol{R}$ R muscles. A) Representative EMG records from pectoralis major muscles during cold

212 exposure showing continuous low-intensity activity in the XX individual and frequent bursts

213 of high-intensity activity in the RR individual. Summary data of the burst rate (B), mean

214 EMG signal frequency $(\mathrm{MnF} ; \mathrm{C})$, and amplitude (RMS, root mean square; D). Plots show

215 values for each RR (black circles) and XX (red circles) individual and mean \pm SEM.

216 Statistical differences between RR and XX individuals were assessed with unpaired t-test. 
220 In conclusion, $\alpha$-actinin-3 deficient humans show improved cold tolerance during cold water

221 immersion, which is associated with a shift towards more slow-twitch type I MyHC. During

222 cold exposure, motor neuron activation occurs mainly as an effective heat-generating increase

223 in muscle tone rather than overt shivering ${ }^{4}$. During prolonged cold exposure, the improved

224 heat generation via increased muscle tone suggests that XX individuals would consume less

225 energy and be less susceptible to developing muscle fatigue compared to individuals that

226 express $\alpha$-actinin-3 (RR), providing an evolutionary survival advantage for XX individuals.

227 This mechanism provides an explanation for the increase in X-allele frequency as modern

228 humans migrated from Africa to the colder colder Euroasian climate over 50.00 years ago ${ }^{2,12}$.

229 However, cold tolerance is seldom a key issue in modern societies and an energy efficient

230 phenotype can be problematic in the context of a current lifestyle with high caloric intake and

231 reduced physical activity.

234 Acknowledgments

235 We thank Monika Kisieliute and Andreius Subocius for their assistance in genotyping and

236 collection of human muscle biopsies. We also thank Sophie Agius and Alison Burns for their

237 contribution to maintaining the Actn3 KO mouse colony.

\section{Author Contribution}

240 TV, MB, HWe conceived the human study. VLW, TV, MS, NI, HP, NE, MB performed

241 experiments in the human study. VLW, TV, MS, VL, NI, HP, NE, DCA, MB, HWe analysed

242 and intrepreted human data. KNN conceived the Actn3 KO mouse study. PJH, CFT, HWo

243 completed the mouse analyses and RNA-sequencing. VLW, PJH, KNN, HWe drafted the

244 manuscript. All authors provided critical evaulation of the manuscript and approved the final

245 version.

247 Conflict of interest statement

248 V.M.L. is founder, CEO, and shareholder of HepaPredict AB. In addition, V.M.L. discloses 249 consultancy work for EnginZyme AB. 


\section{METHODS}

\section{Participants, mice and ethical approval}

253 Humans. Healthy young (18-40 yrs) males were recruited to participate in the study. Before 254 being included in the study, each participant was informed of the aims, the experimental procedures and the potential risks of the study and signed a written informed consent form consistent with the principles outlined in the Declaration of Helsinki. The study was approved by Kaunas Regional Biomedical Research Ethics Committee (license number BE-2-30).

The participants were moderately physically active $(<2 \mathrm{~h}$ physical exercise / week) and did not participate in any formal physical exercise or sport program. They had not been involved in any temperature manipulation programme or extreme-temperature exposure for at least 3 months. Individuals with any existing medical condition or taking medication that could affect natural thermoregulation were excluded from the study. The physical characteristics of the participants are presented in Supplementary Table 1. Their weight (in kg), body fat percentage (TBF-300 body composition analyser, Tanita, UK Ltd., West Drayton, UK) and height (in $\mathrm{cm}$ ) were measured, and body mass index was calculated. Body surface area (in $\mathrm{m}^{2}$ ) was estimated as previously described using the following formula: body surface are $=128.1 \times$ Weight $^{0.44} \times$ Height $^{0.60}{ }^{29}$. Skinfold thickness (in $\mathrm{mm}$ ) was measured with a skinfold calliper (SH5020, Saehan, Masan, Korea) at 10 sites (chin, subscapular, chest, side, suprailium, abdomen, triceps, thigh, knee and calf) and the mean subcutaneous fat layer thickness was calculated ${ }^{30}$.

Actn3 KO and WT mice. All animal work was carried out in accordance with approval from the Murdoch Children's Research Institute Animal Care and Ethics Committee (Approval No. A760). Animals were housed in a specific pathogen-free environment at a constant ambient temperature of $22{ }^{\circ} \mathrm{C}$ and $50 \%$ humidity on a $12 \mathrm{~h}$ light-dark cycle, with ad libitum access to food and water, unless otherwise specified. Generation of Actn3 KO mice on a C57BL/6J background has been previously reported ${ }^{31}$. Age-matched female WT and Actn3 KO littermates derived from heterozygous Actn3 crosses were used for all animal studies.

\section{Genotyping}

281 Human. DNA was extracted from blood samples using the NucleoSpin Blood kit (Macherey-

282 Nagel, GmbH \& Co. KG, Düren, Germany) according to the manufacturer's protocol. 283 ACTN3 R577X genotype was determined using a PCR-RFLP method as previously described 
285 Mouse. Actn3 WT and KO genotypes were determined using PCR-RFLP using DNA extracted from ear punch biopsies and extracted as outlined previously ${ }^{1}$.

\section{Acute cold exposure}

289 Human cold-water immersion protocol. An intermittent whole-body water immersion 290 cooling protocol was used as previously described ${ }^{33-35}$. All experiments were conducted 291 indoors at the same time of day (from 7:00 a.m. to 11:00 a.m.). The participants refrained 292 from consuming any food for at least $12 \mathrm{~h}$ before the experiment. To standardize the state of 293 hydration and the sensation of thirst, subjects were allowed to drink still water as desired until $29460 \mathrm{~min}$ before the water-immersion session. The experiments were performed at a room 295 temperature of $22{ }^{\circ} \mathrm{C}$ and a relative humidity of $60 \%$. Prior to cold-water immersion, the 296 participants rested for 10-15 min dressed in a T-shirt, swim shorts and socks and baseline 297 ventilation parameters, heart rate, and temperatures were measured during the subsequent 20 298 min. Thereafter, they entered a $14{ }^{\circ} \mathrm{C}$ water bath with only the head above the surface. 299 Individuals stepped out of the bath every $20 \mathrm{~min}$ and rested for $10 \mathrm{~min}$ at room temperature, 300 and then returned to the water bath for the next $20 \mathrm{~min}$ of cold-water immersion. This intermittent whole-body water immersion procedure continued until either the rectal temperature $\left(\mathrm{T}_{\mathrm{re}}\right)$ had decreased to $35.5{ }^{\circ} \mathrm{C}$ or a maximum of $120 \mathrm{~min}$ of cold-water immersion (170 min including the breaks).

Mouse acute thermoneutral and cold exposure. For acute temperature exposure experiments, 12 week old female WT and Actn3 KO mice were singly housed in cages kept at either $30^{\circ} \mathrm{C}$ or $4{ }^{\circ} \mathrm{C}$ as previously published ${ }^{36}$. Briefly, mice housed at thermoneutrality $\left(30^{\circ} \mathrm{C}\right.$ ) were acclimatised at this temperature for $20 \mathrm{~h}$ (with food and water ad libitum) prior to commencement of experiments. Food, water and bedding were removed from coldexposed mice during the $5 \mathrm{~h}$ cold exposure period.

\section{Body temperature measurements}

313 Humans. $\mathrm{T}_{\text {re }}$ was measured throughout the experiment using a thermocouple (Rectal Probe,

314 Ellab, Hvidovre, Denmark; accuracy $\pm 0.1^{\circ} \mathrm{C}$ ) which was inserted by the subjects to a depth 315 of $12 \mathrm{~cm}$ past the anal sphincter. Muscle $\left(\mathrm{T}_{\mathrm{mu}}\right)$ and skin $\left(\mathrm{T}_{\mathrm{sk}}\right)$ temperatures were measured 316 before and at the end of the water immersion session. The $\mathrm{T}_{\mathrm{mu}}$ was measured with a needle 317 microprobe (MKA, Ellab; accuracy $\pm 0.01^{\circ} \mathrm{C}$ ) inserted $\sim 3.5 \mathrm{~cm}$ under the skin covering the 318 lateral gastrocnemius muscle of the right leg. The skin was prepared before each 
intramuscular temperature measurement by shaving and disinfecting with a cotton-wool tampon soaked with medicinal alcohol. The insertion area was marked to ensure the repeatability of the measurement. $\mathrm{T}_{\mathrm{sk}}$ was measured with thermistors taped to the back, the thigh and the forearm (DM852, Ellab; accuracy $\pm 0.1{ }^{\circ} \mathrm{C}$ ), and mean $\mathrm{T}_{\text {sk }}$ was calculated as: $\mathrm{T}_{\mathrm{sk}}=0.5 \mathrm{~T}_{\text {back }}+0.36 \mathrm{~T}_{\text {thigh }}+0.14 \mathrm{~T}_{\text {forearm }}{ }^{37}$.

Mice. Core body temperature was measured by a rectal probe (BAT-12 microprobe thermometer) over a 5 hour period between the times of 8:00 and 14:00. Temperatures were measured at $0,30,60,90,120,180,240$, and $300 \mathrm{~min}$. Body weights were recorded before and after the $5 \mathrm{~h}$ temperature measurement period. At the conclusion of the temperature assessment, all mice were euthanized by cervical dislocation and tissues were collected for further analysis.

\section{Human functional measurements}

333 Spirometry and heart rate measurement. A mobile spirometry system (Oxycon Mobile, 334 Jaeger/VIASYS Healthcare, Hoechberg, Germany) was used to measure $\mathrm{VO}_{2}$ and $\mathrm{VCO}_{2}$ on a breath-by-breath basis. Automatic calibration of the gas analyser and delay time were performed before measurements as described by the manufacturer, i.e. a calibration gas at $180 \mathrm{kPa}\left(15.2 \% \mathrm{O}_{2}, 5.02 \% \mathrm{CO}_{2}\right.$, and $\left.79.62 \% \mathrm{~N}_{2}\right)$ was supplied to attain gain, offset, and delay times within $1 \%$. Heart rate was measured throughout the experiment with a heart rate monitor (S-625X, Polar Electro, Kempele, Finland).

EMG measurement of thermoregulatory muscle activation. Heat-generating muscle activation is pronounced in the chest region and we therefore measured EMG signals in the pectoralis major muscle during cold-water immersion ${ }^{38,39}$. After careful preparation of the skin (shaving, abrading, and cleaning with alcohol wipes) to obtain a low impedance $(<10$ kOhm), a surface EMG sensor (SX230W, Biometrics Co., Ltd., Gwent, UK) with integrated bipolar $\mathrm{Ag}-\mathrm{AgCl}$ electrodes $(10 \mathrm{~mm}$ diameter, $20 \mathrm{~mm}$ centre-to-centre distance) and differential amplifier (gain 1000, input impedance $100 \mathrm{M} \Omega$, an input noise $<5 \mu \mathrm{V}$, common mode rejection ratio higher than $96 \mathrm{~dB}$ ) was placed on the right pectoralis major muscle. The ground electrode (R206, Biometrics Co., Ltd.) was positioned on the wrist of the right hand. The EMG sensor and ground electrode were connected to a portable data acquisition unit (DataLog P3X8, Biometrics Co., Ltd.) Before measurements, the channel sensitivity was set to $3 \mathrm{~V}$ and the excitation output to $4600 \mathrm{mV}$ as recommended by the manufacturer. EMG 
353

354

355

356

357

358

359

360

361

362

363

364

365

366

367

368

369

370

371

372

373

374

375

376

377

378

379

380

381

382

383

384

385

386

signals were digitized and files were stored on a computer for subsequent analyses of the mean frequency $(\mathrm{MnF}$, in $\mathrm{Hz})$ and root mean square (RMS, in $\mathrm{mV}$ ) using a dedicated software (Biometrics DataLOG, Gwent, UK) and manual analysis of the rate of burst activity.

\section{Human protein analyses}

Muscle biopsies. Prior to cold-water immersion, biopsies from the vastus lateralis muscle were collected from a subgroup of RR $(n=11)$ and XX $(n=8)$ individuals. The biopsy site was cleaned with alcohol and anesthetized locally. After making a small skin cut with a scalpel tip, a biopsy needle was inserted perpendicular to the muscle fibers and biopsies were collected with and automatic biopsy device (Bard Biopsy Instrument, Bard Radiology, Covington, USA). After collection, muscle biopsies were snap frozen in liquid nitrogen and stored at $-80^{\circ} \mathrm{C}$ until analysis. The skin cut was cleaned and closed with wound closure strips. Biopsies were not collected post cold exposure.

Whole muscle homogenate preparation. Whole muscle homogenate was prepared from frozen muscle biopsies for western blots, analysis of MyHC isoform composition, and immunoprecipitation (IP) experiments. Approximately $15 \mathrm{mg}$ of frozen muscle was weighed and homogenized on ice (1:20 w/v) in HEPES lysis buffer (20 mM HEPES, $150 \mathrm{mM} \mathrm{NaCI}, 5$ mM EDTA, $25 \mathrm{mM} \mathrm{KF}, 5 \%$ Glycerol, $1 \mathrm{mM} \mathrm{Na}_{3} \mathrm{VO}_{4}, 0.5 \%$ Triton, $\mathrm{pH}$ 7.6) with Protease Inhibitor (\#11836145001, Roche, 1 tablet per $50 \mathrm{ml}$ ). After this stage, $70 \mu \mathrm{L}$ was diluted to $33 \mu \mathrm{g}$ wet weight muscle $\mu \mathrm{l}^{-1}$ using $3 \times$ SDS denaturing solution $(0.125 \mathrm{M}$ Tris-HCI, $10 \%$ glycerol, 4\% SDS, $4 \mathrm{M}$ urea, 10\% 2-mercaptoethanol and 0.001\% Bromophenol Blue, pH 6.8). Finally, samples were further diluted to $2.5 \mu \mathrm{g}$ wet weight muscle $\mu 1^{-1}$ with $1 \times \mathrm{SDS}$ solution $(3 \times$ SDS denaturing solution diluted 2:1 with $1 \times$ Tris. $\mathrm{Cl}(\mathrm{pH} 6.8))$. A small amount of undiluted homogenate was taken from each RR participant to make a calibration curve included on every gel for western blotting. The remaining undiluted homogenate was used for IP.

Single fiber collection, fiber-typing and pooling. Approximately $10 \mathrm{mg}$ of muscle was freeze dried for $24 \mathrm{~h}$. Biopsies were left in a desiccator in drying pearls (Sigma) at room temperature for $60 \mathrm{~min}$ and then placed in $-20{ }^{\circ} \mathrm{C}$ for long-term storage. Between 40-60 segments of single fibers were collected from each muscle biopsy and placed in $12 \mu \mathrm{l}$ of $1 \times$ SDS denaturing solution, once collected fibers were kept at room temperature for $60 \mathrm{~min}$ and then stored in $-80{ }^{\circ} \mathrm{C}$ until dot blotting. 
Each single fiber segment was fiber-typed using the dot-blotting method as previously described ${ }^{40}$. Briefly, a PVDF membrane was activated in $96 \%$ ethanol for $120 \mathrm{~s}$ and then activated in transfer buffer containing $20 \%$ methanol for 120 s. Following activation, $1 \mu 1$ from each single fiber tube was spotted on to a membrane. Once the membrane had dried, membranes were reactivated in ethanol (120 s) and transfer buffer $(120 \mathrm{~s})$, membranes were washed in $\times 1$ Tris-buffered saline-Tween (TSBT) and then blocked for $\sim 10 \mathrm{~min}$ in 5\% blocking buffer $($ Bio-Rad) in $1 \times$ TBST. The membrane was then incubated in primary antibody overnight at $4{ }^{\circ} \mathrm{C}$ with $2 \mathrm{~h}$ at room temperature . After washing and incubation with a secondary antibody and $1 \times$ TBST washes the membrane was coated with chemiluminescent substrate (Clarity Max ECL substrate, Bio-Rad) and imaged on Chemidoc MP (Bio-Rad).

In this study, two membranes were prepared simultaneously from each fiber segment. These membranes were probed either for MyHC II (mouse, monoclonal IgG,A4.74,

400 Developmental Studies Hybridoma Bank (DSHB)) or for MyHC I (mouse, monoclonal IgM, A4.840, DSHB) diluted 1:200 in blocking buffer in PBS (LiCOR Biosciences) 1:1 v/v in 1x TBST. Single fiber segments of the same fiber type from each muscle biopsy were subsequently pooled into a single tube and frozen at $-80{ }^{\circ} \mathrm{C}$ until western blotting. Each pool ranged between 4-15 fibers. Only fiber segents that were identified either as MyHC II or MyHC I were used in subsequent analyses.

Western blotting. Proteins of whole homogenates and pools of single fiber segments were separated on either 4-15\% TGX stain-free gels or for analysis of SLN, 16.5\% Tris Tricine gels. TGX stain-free gels had total protein visualized prior to transfer and analyzed on Image Lab software (Image Lab 6.0, Bio-Rad) ${ }^{41}$. Protein was wet transferred to PVDF or nitrocellulose membrane (SLN) for $1 \mathrm{~h}$. Following transfer, tris-tricine gels were stained

412 (Coomassie Brilliant Blue R-250, Bio-Rad) for $2 \mathrm{~h}$ at room temperature and de-stained (40\% 413 methanol, 10\% acetic acid) for $2 \times 1 \mathrm{~h}$ washes at room temperature and then stored overnight 414 in MilliQ $\mathrm{H}_{2} \mathrm{O}$ before being visualized for myosin bands on a Chemi Doc MP (Biorad). 415 Membranes were blocked at room temperature for $2 \mathrm{~h}$ using LI-COR blocking buffer with 416 TBS (LI-COR Biosciences). After blocking, membranes were incubated in primary antibody 417 overnight at $4^{\circ} \mathrm{C}$ and $2 \mathrm{~h}$ at room temperature. Primary antibody details are as follows: 418 SERCA2a (1:5000, rabbit, A010-20, Badrilla), SERCA1 (1:1000, mouse, CaF2-5D2, 419 DHSB), CSQ1\&2 (1:1000, mouse, MA3-913, Thermofisher), CSQ2 (1:1000, rabbit, ab3516, 420 Abcam), ACTN3 (1:0000, rabbit, ab68204, Abcam), SLN (1:1000, rabbit, ABT13, Merck 
Millipore), MyHC II (1:200, mouse IgG,A4.74, DHSB), MyHC I (1:200, Mouse, IgM, A4.840, DSHB). and actin (1:1000 rabbit, ab1801, Abcam). All antibodies were diluted in LI-COR blocking buffer in PBS (LI-COR Biosciences) $1: 1 \mathrm{v} / \mathrm{v}$ with $1 \times$ TBST. After incubation in primary antibody, membranes were washed in $1 \times$ TBST, incubated in secondary antibody (1:20,000, IRDye 680-conjugated donkey anti-mouse IgG and IRDye 800-conjugated donkey anti-rabbit IgG (926-68,072, 926-32,213), LI-COR Biosciences) and immunoreactive bands were visualized using infrared fluorescence (IR-Odyssey scanner, LICOR Biosciences). Band density was analyzed using Image Studio Lite v 5.2 (LI-COR Biosciences). During data analysis, the density of each sample for a given protein was expressed relative to the calibration curve and then normalized to the total protein of each respective lane. SLN in homogenate was normalized against actin, which was not different between RR and XX individuals. Single fiber SLN was normalized against Coomassie stain. The same calibration curve was used across all gels and data are expressed relative to the average of the RR subjects on each gel, which was set to 1.0 .

Myosin heavy chain composition. MyHC composition was determined by electrophoresis using a protocol adapted from Mizunoya et al. ${ }^{42}$. The whole muscle homogenates were diluted 2.5 times in MilliQ $\mathrm{H}_{2} \mathrm{O}$ and then in $2 \times$ sample buffer containing $100 \mathrm{mM}$ DTT, 4\% w/v SDS, $0.16 \mathrm{M}$ Tris-HCl (pH 6.8), 43\% v/v glycerol and $0.2 \%$ bromophenol blue. $10 \mu 1$ of each sample was loaded on a separating gel consisting of $100 \mathrm{mM}$ glycine, 35\% v/v glycerol, $200 \mathrm{mM}$ Tris-HCl $(\mathrm{pH} \quad 8.8), \quad 0.35 \% \quad$ w/v $\quad$ SDS, $8.5 \% \quad$ w/v acrylamide $-N, N^{\prime}-$ methlyenebisacrylamide (99:1), $0.1 \% \mathrm{w} / \mathrm{v}$ ammonium persulfate and $0.05 \% \mathrm{v} / \mathrm{v} N, N, N^{\prime}, N^{\prime}-$ tetramethylethylenediamine. The stacking gel consisted of $10 \% \mathrm{v} / \mathrm{v}$ glycerol, $70 \mathrm{mM}$ Tris$\mathrm{HCl} \quad\left(\mathrm{pH}\right.$ 6.8), 4 mM EDTA, $0.34 \%$ w/v $\quad$ SDS, $4 \% \quad \mathrm{w} / \mathrm{v}$ acrylamide- $N, N^{\prime}-$ methlyenebisacrylamide (99:1), 0.1\% w/v ammonium persulfate and $0.05 \% \mathrm{v} / \mathrm{v} N, N, N^{\prime}, N^{\prime}-$ tetramethylethylenediamine. After adding lower (0.05 M Tris Base, $75 \mathrm{mM}$ glycine, $0.05 \%$ w/v SDS) and upper running buffer $(6 \times$ concentrated lower running buffer with $0.12 \%$ v/v 2 mercaptoethanol), electrophoresis was run at $4{ }^{\circ} \mathrm{C}$ for $40 \mathrm{~min}$ at $10 \mathrm{~mA}$ and then for $22 \mathrm{~h}$ and $20 \mathrm{~min}$ at $140 \mathrm{~V}$. After electrophoresis, gels were stained with the SilverXpress Silver Staining Kit (Invitrogen) according to the manufacturer's instruction. Bands were analyzed using ImageJ software. 
manufacturer's instructions. The lysates were centrifuged at $700 \mathrm{~g}$ and $4{ }^{\circ} \mathrm{C}$ for $10 \mathrm{~min}$ and the protein concentration of the supernatant was determined with the Bio-Rad Protein Assay (\#500-0006). The samples were then diluted to $0.5 \mu \mathrm{g}$ protein per $\mu 1$ and $400 \mu 1$ of each sample was added to the antibody-bead complex. Samples were incubated overnight at $4{ }^{\circ} \mathrm{C}$ under gentle rotation. After incubation, samples gently washed four times with HEPES buffer. Samples were placed on a magnet rack for removal of buffer. To remove separate peptides from the beads, $50 \mu 1$ Laemmli buffer (Bio-Rad) with 5\% 2-mercaptoethanol were added and samples were heated for $5 \mathrm{~min}$ at $95{ }^{\circ} \mathrm{C}$. To remove the beads from the solution, tubes were placed on a magnet and the solution was transferred to fresh tubes. Samples (10 $\mu \mathrm{g} /$ well) were loaded on precast 4-12\% Bis-Tris gels (NuPAGE, Invitrogen) and run for $1 \mathrm{~h}$ at $150 \mathrm{~V}$. Proteins were transferred to PVDF membranes for $3 \mathrm{~h}$ on ice. After blocking in blocking buffer (LI-COR) and TBS-T for $1 \mathrm{~h}$, membranes were incubated overnight with anti-RyR1 (1:5000, mouse, ab2868, Abcam) and anti-FKBP12 (1:1000 rabbit, ab2918, Abcam) antibodies. After washing with TBS-T, membranes were incubated in secondary antibody (1:20,000, IRDye 680-conjugated donkey anti-mouse IgG and IRDye 800conjugated donkey anti-rabbit IgG (926-68,072, 926-32,213, LI-COR Biosciences) for $1 \mathrm{~h}$ at room temperature. Membranes were washed three times with TBS-T and bands were visualized using an infrared fluorescence scanner (IR-Odyssey, LI-COR Biosciences). Band densities were analyzed with Image Studio Lite v 5.2 software (LI-COR). Data are expressed as ratios of FKBP12/RyR1 relative to the group mean of the RR group, which was set to 1.0.

\section{Human Proteomics Analysis}

Protein extraction and solubilization. Vastus lateralis muscle biopsies were homogenized in $16 \mu \mathrm{g} / \mathrm{ml}$ PBS and briefly centrifuged. After discarding the supernatant, $200 \mu 1$ lysis buffer (8M Urea, 1\% SDS, $50 \mathrm{mM}$ Tris $\mathrm{pH} 8.5$, Roche protease and phosphatase inhibitor) were added to the pellet and samples were vortexed and sonicated on ice. Cell lysates were then centrifuged for $10 \mathrm{~min}$ at $4{ }^{\circ} \mathrm{C}$ and $15,000 \mathrm{rpm}$ and cleared lysates were transferred to new tubes. The extracted proteins were precipitated with chilled acetone $(1: 4 \mathrm{vol})$ at $-20{ }^{\circ} \mathrm{C}$ overnight and then centrifuged for $20 \mathrm{~min}$ at $14,000 \mathrm{~g}$ at $4{ }^{\circ} \mathrm{C}$. The protein pellets were dissolved in $40 \mu \mathrm{l}$ of $8 \mathrm{M}$ urea and a $3 \mu \mathrm{l}$ aliquot of each sample was diluted 10-fold to BCAassay. A volume equivalent of $25 \mu \mathrm{g}$ of proteins from each sample were adjusted to $43 \mu \mathrm{l}$ with water and $5 \mu \mathrm{l}$ of $1 \mathrm{M}$ ammonium bicarbonate (AmBic) was added. 
In solution digestion and TMT-labeling. Proteins were reduced with adding $1.5 \mu 1$ of 200 $\mathrm{mM}$ dithiothreitol (DTT, Sigma) in $500 \mathrm{mM}$ AmBic and incubated at $37{ }^{\circ} \mathrm{C}$ for $1 \mathrm{~h}$ with shaking at $400 \mathrm{rpm}$. Alkylation was performed with adding $1.5 \mu \mathrm{l}$ of $66 \mathrm{mM}$ iodoacetamide (Sigma) in $500 \mathrm{mM} \mathrm{AmBic} \mathrm{at} \mathrm{room} \mathrm{temperature} \mathrm{for} 30 \mathrm{~min}$ with shaking at $400 \mathrm{rpm}$. Thereafter $1 \mu \mathrm{g}$ of sequencing grade modified Trypsin (Promega) was added to each sample (1:33 trypsin:protein) and incubated for $16 \mathrm{~h}$ at $37{ }^{\circ} \mathrm{C}$. The digestion was stopped by adding of formic acid at final concentration of $5 \%$ and incubating the solution for 20 min at $37{ }^{\circ} \mathrm{C}$. Then the samples were cleaned on a C18 Hypersep plate (Thermo Scientific), dried using a Speedvac and re-suspended in $70 \mu \mathrm{l}$ of $50 \mathrm{mM}$ triethylammonium bicarbonate (TEAB) buffer and $30 \mu \mathrm{l}$ of TMT-10plex (Thermo Scientific) reagent was added in dry acetonitrile (ACN) following incubation for $2 \mathrm{~h}$ at room temperature with shaking at $550 \mathrm{rpm}$. Labeling reaction was quenched with $11 \mu \mathrm{l}$ of 5\% hydroxylamine. Labeled samples were combined and dried on Speedvac. Following a cleaning on StageTip C18 $20 \mu \mathrm{l}$ of the combined samples were dissolved in $0.1 \%$ formic acid and $2 \% \mathrm{ACN}$.

PRLC-MS/MS analysis. Chromatographic separation of peptides was achieved using a 50 Scientific). Approximately $1.3 \mu \mathrm{g}$ peptides were loaded onto the column in a volume of $2 \mu 1$ and then eluted at a $300 \mathrm{nl} / \mathrm{min}$ flow rate for $180 \mathrm{~min}$ at a linear gradient from $4 \%$ to $26 \%$ ACN in $0.1 \%$ formic acid. Orbitrap Q Exactive plus mass spectrometer (Thermo Scientific) analyzed the eluted peptides that were ionized with electrospray ionization. The survey MS spectrum was acquired at the resolution of 140,000 in the range of $\mathrm{m} / \mathrm{z} 350-1600$. MS/MS data were obtained with a higher-energy collisional dissociation (HCD) for ions with charge $\mathrm{z}=2-3$ at a resolution of $70,000 \mathrm{using} \mathrm{m} / \mathrm{z} 2$ isolation width.

513 Proteomics data analysis. Data was analyzed on Proteome Discoverer v2.2 (Thermo

514 Scientific) identifying protein in SwissProt database and the extracted abundances were 515 further evaluated using an in-house developed $\mathrm{R}$ algorithm calculating fold changes and $P$ values. Data were visualized using Qlucore Omics Explorer (Lund, Sweden).

\section{RNA-sequencing in BAT of Actn3 KO and WT mice.}

519 Brown adipose tissue (BAT) was collected from Actn3 WT and KO mice after $5 \mathrm{~h}$ of core 520 body temperature analyses across the three treatment groups (Thermoneutral, TN, Room521 temperature, RT, and cold exposed, CE). A total of $18 \mathrm{WT}$ and $16 \mathrm{KO}$ mice underwent RNA 
522 sequencing using the Illumina HiSeq 2500 platform as per manufacturer instructions. Raw

523 read data was processed using the Illumina BaseSpace RNA Express application (Illumina 524 Inc. 2016). Briefly, sequencing reads were aligned using STAR ultrafast RNA seq aligner ${ }^{43}$ 525 in the SAM file format ${ }^{44}$, then counted using HTSeq ${ }^{45}$. The resulting genewise count data 526 was analyzed using the R (3.6.0) statistical programming language (R Core Team 2018). 527 Modelling of differential expression was conducted using the voom precision weights 528 approach ${ }^{46}$ in the limma (3.40.6) ${ }^{47}$ package from the Bioconductor (3.9) project.

529

$530 \quad$ Statistics

531 Statistical analyses were performed using GraphPad Prism 8. Unpaired t-tests were used to 532 assess statistical significance between the RR and XX group. Mantel-COX log-rank test was 533 used for comparisons of the effect of cold-water immersion (baseline to end-point). Data are 534 presented as mean \pm SEM and $P \leq 0.05$ was considered statistically significant. 
5371 MacArthur, D. G. et al. Loss of ACTN3 gene function alters mouse muscle

538

539

540

541

542

543

544

545

546

547

548

549

550

551

552

553

554

555

556

557

558

559

560

561

562

563

564

565

566

567

568

569

570

571

572

573

574

575

576

577

578

579

580

581

582

583 metabolism and shows evidence of positive selection in humans. Nat. Genet. 39, 1261-1265 (2007).

2 Friedlander, S. M. et al. ACTN3 allele frequency in humans covaries with global latitudinal gradient. PLoS One 8, e52282 (2013).

3 Haman, F. \& Blondin, D. P. Shivering thermogenesis in humans: Origin, contribution and metabolic requirement. Temperature 4, 217-226 (2017).

4 Lømo, T., Eken, T., Bekkestad Rein, E. \& Nja, A. Body temperature control in rats by muscle tone during rest or sleep. Acta Physiol., e13348 (2019).

5 Mills, M. et al. Differential expression of the actin-binding proteins, alpha-actinin-2 and -3 , in different species: implications for the evolution of functional redundancy. Hum. Mol. Genet. 10, 1335-1346 (2001).

6 North, K. N. et al. A common nonsense mutation results in alpha-actinin-3 deficiency in the general population. Nat. Genet. 21, 353-354 (1999).

7 Quinlan, K. G. et al. Alpha-actinin-3 deficiency results in reduced glycogen phosphorylase activity and altered calcium handling in skeletal muscle. Hum. Mol. Genet. 19, 1335-1346 (2010).

8 Houweling, P. J. et al. Is evolutionary loss our gain? The role of ACTN3 p.Arg577Ter (R577X) genotype in athletic performance, ageing, and disease. Hum. Mutat. 39, 1774-1787 (2018).

9 Pickering, C. \& Kiely, J. ACTN3, morbidity, and healthy aging. Front. Genet. 9, 15 (2018).

10 Lee, F. X., Houweling, P. J., North, K. N. \& Quinlan, K. G. How does alpha-actinin-3 deficiency alter muscle function? Mechanistic insights into ACTN3, the 'gene for speed'. Biochim. Biophys. Acta 1863, 686-693 (2016).

11 Del Coso, J. et al. More than a 'speed gene': ACTN3 R577X genotype, trainability, muscle damage, and the risk for injuries. Eur. J. Appl. Physiol. 119, 49-60 (2019).

12 Amorim, C. E. G., Acuna-Alonzo, V., Salzano, F. M., Bortolini, M. C. \& Hunemeier, T. Differing evolutionary histories of the ACTN3*R577X polymorphism among the major human geographic groups. PloS One 10, e0115449 (2015).

13 Head, S. I. et al. Altered $\mathrm{Ca}^{2+}$ kinetics associated with alpha-actinin-3 deficiency may explain positive selection for ACTN3 null allele in human evolution. PLoS Genet 11, e1004862 (2015).

14 de Meis, L. Role of the sarcoplasmic reticulum $\mathrm{Ca}^{2+}$-ATPase on heat production and thermogenesis. Biosci. Rep. 21, 113-137 (2001).

15 Bal, N. C., Maurya, S. K., Singh, S., Wehrens, X. H. \& Periasamy, M. Increased reliance on muscle-based thermogenesis upon acute minimization of brown adipose tissue function. J. Biol. Chem. 291, 17247-17257 (2016).

16 Nowack, J., Giroud, S., Arnold, W. \& Ruf, T. Muscle non-shivering thermogenesis and its role in the evolution of endothermy. Front. Physiol. 8, 889 (2017).

17 Rowland, L. A., Bal, N. C. \& Periasamy, M. The role of skeletal-muscle-based thermogenic mechanisms in vertebrate endothermy. Biol. Rev. Camb. Philos. Soc. 90, 1279-1297 (2015).

18 Periasamy, M. \& Kalyanasundaram, A. SERCA pump isoforms: their role in calcium transport and disease. Muscle Nerve 35, 430-442 (2007).

19 Schiaffino, S. \& Reggiani, C. Fiber types in mammalian skeletal muscles. Physiol. Rev. 91, 1447-1531 (2011). 
610

611

612

613

614

615

616

617

618

619

620

621

622

623

624

625

626

627

628

629

630

631

632

633

20 Lamboley, C. R., Murphy, R. M., McKenna, M. J. \& Lamb, G. D. Sarcoplasmic reticulum $\mathrm{Ca}^{2+}$ uptake and leak properties, and SERCA isoform expression, in type I and type II fibres of human skeletal muscle. J. Physiol. 592, 1381-1395 (2014).

21 Lamboley, C. R., Murphy, R. M., McKenna, M. J. \& Lamb, G. D. Endogenous and maximal sarcoplasmic reticulum calcium content and calsequestrin expression in type I and type II human skeletal muscle fibres. J. Physiol. 591, 6053-6068 (2013).

22 Mall, S. et al. The presence of sarcolipin results in increased heat production by $\mathrm{Ca}^{2+}-$ ATPase. J. Biol. Chem. 281, 36597-36602 (2006).

23 Aydin, J. et al. Nonshivering thermogenesis protects against defective calcium handling in muscle. FASEB J. 22, 3919-3924 (2008).

24 Zalk, R. et al. Structure of a mammalian ryanodine receptor. Nature 517, 44-49 (2015).

25 Nedergaard, J. \& Cannon, B. Brown adipose tissue as a heat-producing thermoeffector. Handb. Clin. Neurol. 156, 137-152 (2018).

26 Ong, F. J. et al. Recent advances in the detection of brown adipose tissue in adult humans: a review. Clin. Sci. 132, 1039-1054 (2018).

27 Meigal, A. Gross and fine neuromuscular performance at cold shivering. Int. J. Circumpolar Health 61, 163-172 (2002).

28 Blondin, D. P. et al. Inhibition of intracellular triglyceride lipolysis suppresses coldinduced brown adipose tissue metabolism and increases shivering in humans. Cell Metab. 25, 438-447 (2017).

29 Tikuisis, P., Jacobs, I., Moroz, D., Vallerand, A. L. \& Martineau, L. Comparison of thermoregulatory responses between men and women immersed in cold water. $J$. Appl. Physiol. 89, 1403-1411 (2000).

30 McArdle, W. D., Magel, J. R., Gergley, T. J., Spina, R. J. \& Toner, M. M. Thermal adjustment to cold-water exposure in resting men and women. J. Appl. Physiol. Respir. Environ. Exerc. Physiol. 56, 1565-1571 (1984).

31 Houweling, P. J. et al. Exploring the relationship between $\alpha$-actinin-3 deficiency and obesity in mice and humans. Int. J. Obes. 41, 1154-1157 (2017).

32 Venckunas, T. et al. Human alpha-actinin-3 genotype association with exerciseinduced muscle damage and the repeated-bout effect. Appl. Physiol. Nutr. Metab. 37, 1038-1046 (2012).

33 Brazaitis, M. et al. Two strategies for response to 14 degrees C cold-water immersion: is there a difference in the response of motor, cognitive, immune and stress markers? PLoS One 9, e109020 (2014).

34 Brazaitis, M. et al. Brief rewarming blunts hypothermia-induced alterations in sensation, motor drive and cognition. Front. Physiol. 7, 592 (2016).

35 Solianik, R., Skurvydas, A., Mickeviciene, D. \& Brazaitis, M. Intermittent wholebody cold immersion induces similar thermal stress but different motor and cognitive responses between males and females. Cryobiology 69, 323-332 (2014).

36 Knights, A. J. et al. Eosinophil function in adipose tissue is regulated by Krüppel-like factor 3 (KLF3). Nat. Commun. 11, 2922 (2020).

37 Lenhardt, R. \& Sessler, D. I. Estimation of mean body temperature from mean skin and core temperature. Anesthesiology 105, 1117-1121 (2006).

38 van Ooijen, A. M., van Marken Lichtenbelt, W. D., van Steenhoven, A. A. \& Westerterp, K. R. Cold-induced heat production preceding shivering. Br. J. Nutr. 93, 387-391 (2005).

39 Blondin, D. P. et al. Contributions of white and brown adipose tissues and skeletal muscles to acute cold-induced metabolic responses in healthy men. J. Physiol. 593, 701-714 (2015). 
63440 Christiansen, D. et al. A fast, reliable and sample-sparing method to identify fibre

635

636 types of single muscle fibres. Sci. Rep. 9, 6473 (2019).

637

638

639

41 Lamboley, C. R., Wyckelsma, V. L., McKenna, M. J., Murphy, R. M. \& Lamb, G. D.

41 Lamboley, C. R., Wyckelsma, V. L., McKenna, M. J., Murphy, R. M. \& Lamb, G. D. $\mathrm{Ca}^{2+}$ leakage out of the sarcoplasmic reticulum is increased in type I skeletal muscle fibres in aged humans. J. Physiol. 594, 469-481 (2016).

640

641

642

643

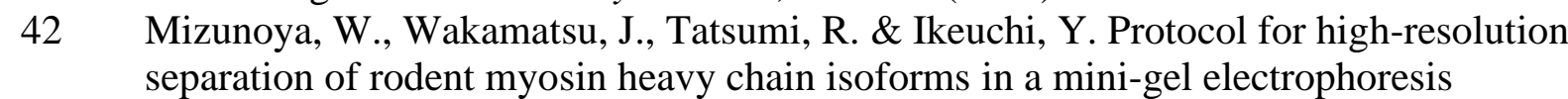
system. Anal. Biochem. 377, 111-113 (2008).

43 Dobin, A. et al. STAR: ultrafast universal RNA-seq aligner. Bioinformatics 29, 15-21

$64444 \mathrm{Li}$, H. et al. The Sequence Alignment/Map format and SAMtools. Bioinformatics 25, 645 2078-2079 (2009).

64645 Anders, S., Pyl, P. T. \& Huber, W. HTSeq--a Python framework to work with high-

647

648 throughput sequencing data. Bioinformatics 31, 166-169 (2015).

649 Law, C. W., Chen, Y., Shi, W. \& Smyth, G. K. voom: Precision weights unlock linear

650 model analysis tools for RNA-seq read counts. Genome Biol. 15, R29 (2014).

651

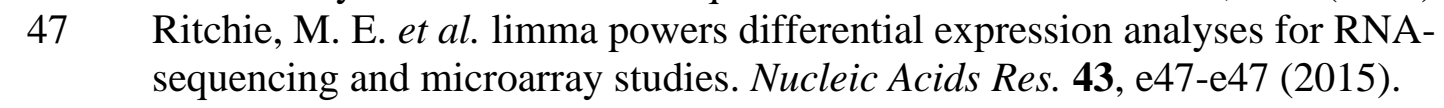




\section{SUPPLEMENTARY RESULTS}

655 Table 1 shows physical characteristics and baseline temperatures of individuals in the RR and 656 XX groups. RR subjects were slightly younger than XX subjects, whereas no statistically 657 significant differences were observed for the other measured physical properties or baseline 658 temperatures.

659

660 Supplementary Table 1. Physical characteristics and baseline temperatures of the 661 individuals in the RR and XX groups.

\begin{tabular}{lll}
\hline & \multicolumn{2}{l}{ ACTN3 genotype groups } \\
\cline { 2 - 3 } & RR (n=27) & XX (n=15) \\
\hline Age, yrs & $22 \pm 1$ & $28 \pm 2 *$ \\
Height, cm & $181 \pm 1$ & $183 \pm 2$ \\
Body weight, $\mathrm{kg}$ & $77.2 \pm 2.5$ & $81.2 \pm 3.5$ \\
Body surface area, ${ }^{2}{ }^{*}$ & $1.96 \pm 0.03$ & $2.01 \pm 0.05$ \\
Mean skinfold thickness, $\mathrm{mm}$ & $12.1 \pm 0.9$ & $14.5 \pm 1.3$ \\
Body mass index, $\mathrm{kg} \cdot \mathrm{m}^{-2}$ & $23.6 \pm 0.6$ & $24.3 \pm 0.7$ \\
Body fat, $\%$ & $15.7 \pm 1.0$ & $17.9 \pm 1.1$ \\
Rectal T, ${ }^{\circ} \mathrm{C}$ & $36.9 \pm 0.1$ & $36.8 \pm 0.1$ \\
Calf muscle $\mathrm{T},{ }^{\circ} \mathrm{C}$ & $35.6 \pm 0.1$ & $35.2 \pm 0.2$ \\
Skin $\mathrm{T},{ }^{\circ} \mathrm{C}$ & $32.1 \pm 0.1$ & $31.8 \pm 0.2$ \\
\hline Values are &
\end{tabular}

Values are mean \pm SEM. $* P<0.05$ in unpaired t-test. 
A

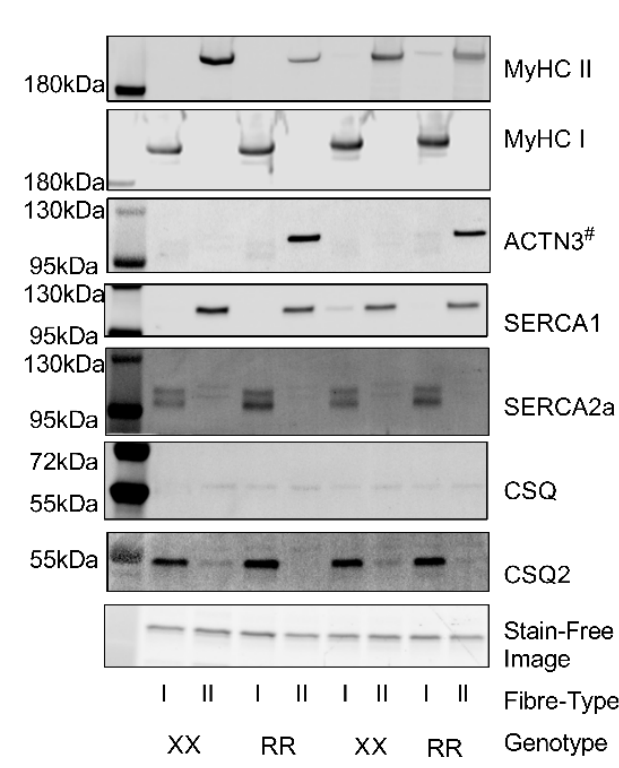

B

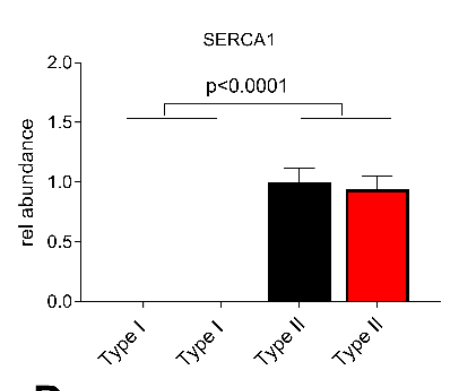

D

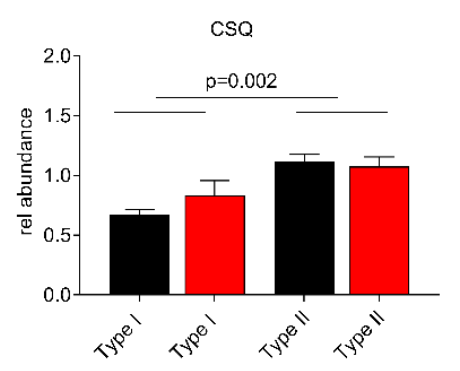

C

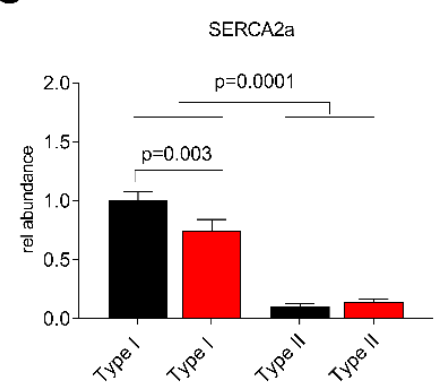

E

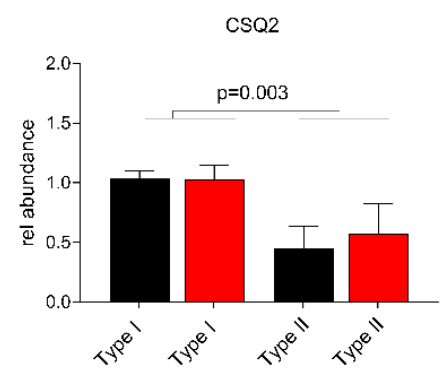

664

665

\section{Supplementary Figure 1.}

667 Representative blots (A) and summary data of pooled single fiber expression of SERCA1 (B),

668 SERCA2a (C), CSQ (D), and CSQ2 (E). Fibers were identified as either Type I (MyHC I) or

669 Type II (MyHC II). ACTN3 has a similar molecular weight as both SERCA1 and 2a, hence it

670 was not possible to probe for ACTN3 on the same membrane as SERCA1 and 2 and an extra

671 gel was run for ACTN3 (marked with \#). Stain free images show the actin prior to transfer,

672 which was used as a loading control. For each single fiber pool, proteins were normalized

673 against their own calibration curve $(\sim 5-40 \mu \mathrm{g}$ wet weight protein) and total protein and

674 expressed relative to the mean of the RR fibers, which was set to 1; proteins mainly

675 expressed in Type I fibers were expressed relative to the mean of the RR Type I fibers and

676 vice versa for proteins mainly expressed in Type II fibers. Data are presented as mean \pm

677 SEM. Differences between RR and XX were determined by one-way ANOVA with Tukey's

678 post hoc test.

679 

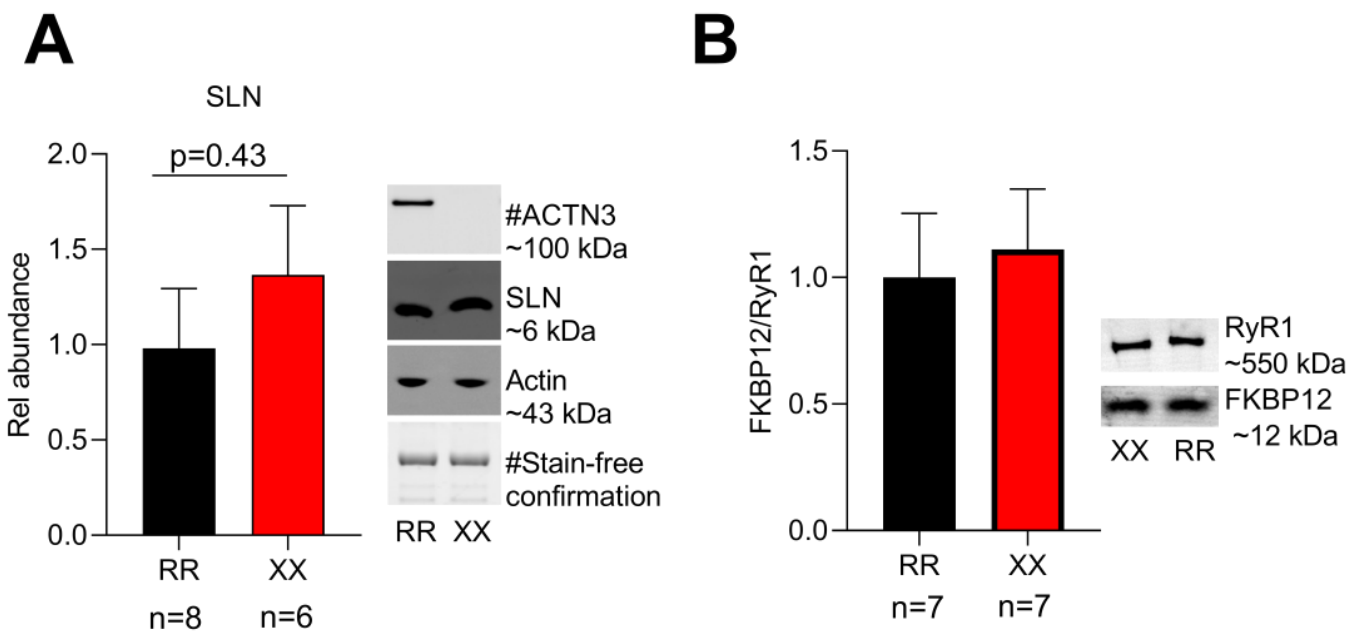

680

681

682 Supplementary Figure 2.

683 Summary data (mean \pm SEM) and representative whole muscle homogenate western blots of

684 SLN (A) and FKBP12 associated with RyR1 (B). Band intensities in A were normalized to 685 their respective actin stain-free loading controls; \# in $\mathbf{A}$ indicates a separate gel from that of 686 SLN (qualitatively similar results obtained with actin on same gel used as loading control). 687 Data are expressed relative to the mean value of the RR group, which was set to 1.0. No 688 statistical difference $(P>0.05)$ in SLN expression or FKBP12 associated with RyR1 between 689 RR and XX individuals were observed with unpaired t-test. 
691
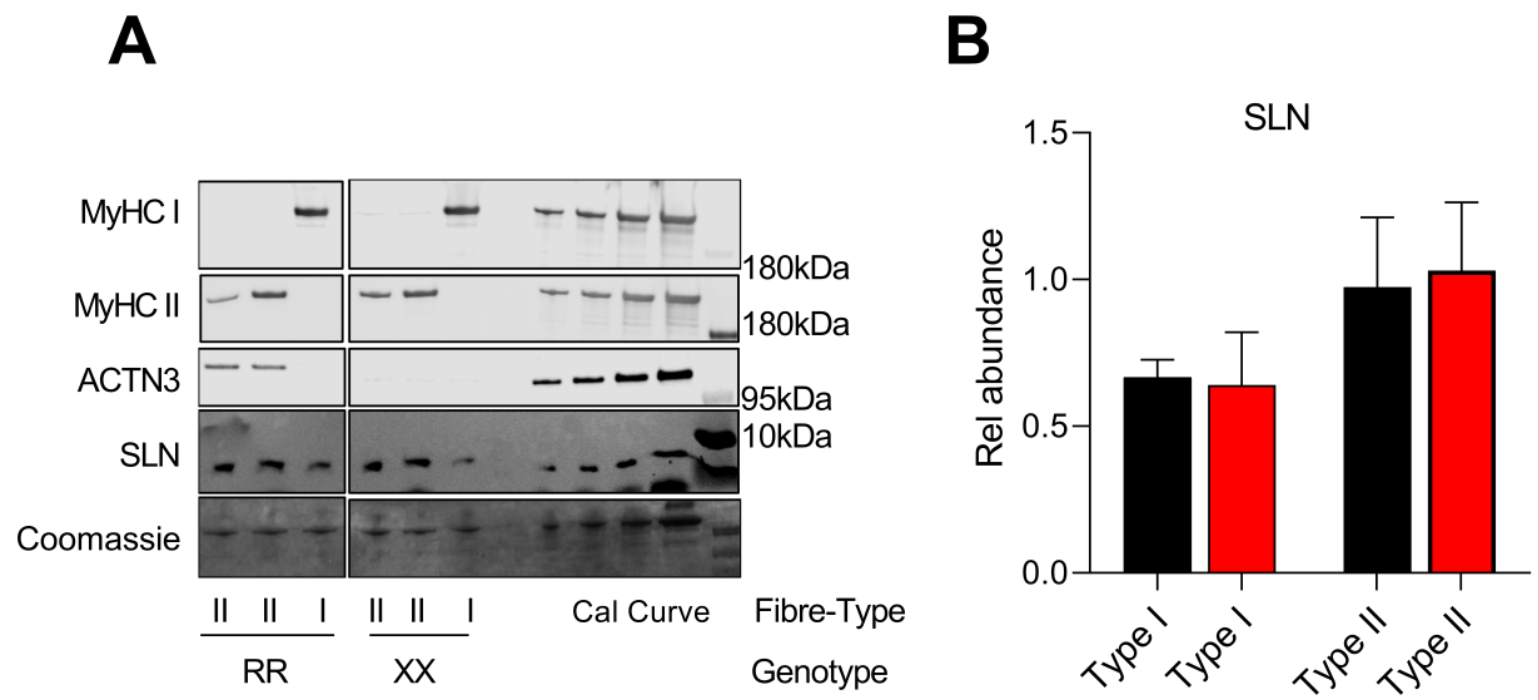

692

693

\section{Supplementary Figure 3.}

695 A) Representative blots for analysis of SLN protein expression in pooled single fibers. SLN

696 was run on a $16.5 \%$ Tris Glycine Gel and Coomassie stained after transfer for loading

697 control. The two MyHC isoforms and ACTN3 were run on two different gels, loaded

698 identical to those described for SLN. Fibers are identified as either Type I (MyHC I), Type II

699 (MyHC II). B) For each single fiber pool, SLN was normalized against its own calibration

700 curve and protein content and expressed relative to the mean of the RR MyHC II, which was

701 set to 1. Data are shown as mean \pm SEM. 